\title{
Applications of Discrete Wavelet Analysis for Predicting Internal Quality of Cherry Tomatoes using VIS/NIR Spectroscopy
}

\author{
Ghiseok Kim1, Dae-Yong Kim², Geon Hee Kim, Byoung-Kwan $\mathrm{Cho}^{2}$ * \\ ${ }^{1}$ Center for Analytical Instrumentation Development, Korea Basic Science Institute, 169-148 Gwahak-ro, \\ Yuseong-gu, Daejeon, Korea \\ ${ }^{2}$ Department of Biosystems Machinery Engineering, Chungnam National University, \\ 99 Daehak-ro, Yuseong-gu, Daejeon, Korea
}

Received: February $18^{\text {th }}, 2013$; Revised: February $28^{\text {th }}, 2013$; Accepted: February $28^{\text {th }}, 2013$

\section{Abstract}

Purpose: This study evaluated the feasibility of using a discrete wavelet transform (DWT) method as a preprocessing tool for visible/near-infrared spectroscopy (VIS/NIRS) with a spectroscopic transmittance dataset for predicting the internal quality of cherry tomatoes. Methods: VIS/NIRS was used to acquire transmittance spectrum data, to which a DWT was applied to generate new variables in the wavelet domain, which replaced the original spectral signal for subsequent partial least squares (PLS) regression analysis and prediction modeling. The DWT concept and its importance are described with emphasis on the properties that make the DWT a suitable transform for analyzing spectroscopic data. Results: The $\mathrm{R}^{2}$ values and root mean squared errors (RMSEs) of calibration and prediction models for the firmness, sugar content, and titratable acidity of cherry tomatoes obtained by applying the DWT to a PLS regression with a set of spectra showed more enhanced results than those of each model obtained from raw data and mean normalization preprocessing through PLS regression. Conclusions: The developed DWT-incorporated PLS models using the db5 wavelet base and selected approximation coefficients indicate their feasibility as good preprocessing tools by improving the prediction of firmness and titratable acidity for cherry tomatoes with respect to $\mathrm{R}^{2}$ values and RMSEs.

Keywords: Visible and near-infrared spectroscopy (VIS/NIRS), Discrete wavelet transform (DWT), Partial least squares (PLS), Internal quality, Cherry tomato

\section{Introduction}

In the postharvest process of the agricultural industry, one difficulty in the production of high-quality goods such as vegetables and fruits is evaluating their quality in a manner that is nondestructive. Various nondestructive measurement methods with highly enhanced ultrasound, $\mathrm{X}$-ray, electromagnetic, or optical analysis techniques have been developed to evaluate the internal quality of fruits or vegetables. As a powerful analytical tool, visible/ near-infrared spectroscopy (VIS/NIRS) has been gaining

\footnotetext{
*Corresponding author: Byoung-Kwan Cho

Tel: +82-42-821-6715; Fax: +82-42-823-6246

E-mail: chobk@cnu.ac.kr
}

popularity over the last decade. This optical sensor-based technique collects the spectroscopic information of target objects with hundreds of continuous wavebands. Because VIS/NIRS as a measurement method is nondestructive, low cost, enables fast data analysis, and follows simple measuring procedures, in addition to its intrinsic characteristics, which contain detailed physical and chemical information on the target objects, its applications can be found not only in agriculture but also in many other fields, including food science, military, biological, and biomedical areas.

Chemometrics is considered to be the final analysis method for preprocessed spectral data obtained by spectroscopy. Several chemometric methods have been 
used for analyzing spectral data. The principal componentstepwise regression algorithm (PCR-SRA) is widely used for the quantitatively analyzing components of materials (Qi et al., 1999). The PCR can effectively compress the dimensions of the original independent variables by relating the original independent variables to new reduced dimension independent variables. However, the degree of correlation between the original independent variables and newly mapped dependent variable by reduced dimension independent variables may decrease, which in practice results in low prediction precision with the PCR. Partial least squares (PLS) regression is also employed as a major regression technique for multivariate data (Brereton, 2000). One important practical aspect of PLS is that it takes into account errors both in the concentration estimates and in the spectra. Therefore, a model established by PLS is bound to be affected by an outlier, and avoiding and solving this problem are essential when employing a PLS model.

In the case of analyzing spectral data for agricultural samples, fluctuating background noise and the vast amount of overlapping information may hamper the accurate evaluation of their quality. Therefore, preprocessing the raw spectral data is essential for noise reduction and eliminating unnecessary environmental influences or interferences. Some popular preprocessing techniques, including a derivative method (Karstang and Kvalheim, 1991), Fourier filtering (Arnold and Small, 1990), and orthogonal signal correction (Svensson et al., 2002), have been used to remove background signals and noise from spectral data. Recently, wavelet transform methods have been widely used as alternative preprocessing tools in VIS/NIR spectral analysis (Walczak and Massart, 1997; Jetter et al., 2000). Studies have demonstrated that a discrete wavelet transform (DWT) is an excellent tool for fine- and coarse-scale spectral feature separation in hyperspectral data (Bruce and Koger, 2002; Zhang et al., 2005). Thus, the DWT is expected to be a valuable means of preprocessing data obtained from VIS/NIRS.

The objectives of our study were to incorporate the DWT as a tool for preprocessing data obtained from VIS/NIRS for improving the prediction accuracy of physical and chemical constituent concentrations of cherry tomatoes, and to compare the results of DWTpreprocessed PLS models with those of PLS models that use raw data and mean normalization preprocessing methods.

\section{Materials and Methods}

\section{VIS/NIR spectroscopic measurement}

The VIS/NIRS measurement and analysis process used in this study consisted of four main steps: (1) The transmittance spectra of target samples were measured, and their physical and chemical constituents were measured using conventional destructive methods; (2) the transmittance spectra were manipulated using various preprocessing methods, including DWT analysis and mean normalization; (3) a subset of spectral data was used to develop a calibration model using PLS regression; and (4) the spectral data that had not been used in the calibration step were used to develop prediction models for estimating the physical and chemical constituents of sample materials.

\section{Discrete wavelet transform analysis}

In spectral analysis, the useful information of unprocessed spectra will often be spread out over many points and can be affected by interference or various types of noise. Thus, the application of linear transforms to spectra can be helpful in concentrating significant information into as few transform variables as possible while separating this information from noise. These transforms not only add any information to the original signal but also simply manipulate the information so that it is represented in a different manner. Therefore, this transformation work will help represent important spectral features when some of its base functions closely resemble the spectral features of interest. It means that the good transform will be accomplished when a base function is well matched to the shapes of important spectral characteristics (McNulty and Mauze, 1998).

In this study, transmittance spectra were considered as one-dimensional, discrete, finite-length signals, and DWT preprocessing was incorporated. Mathematically, the DWT of a finite-length signal involves two types of complementary series: a series of approximation coefficients at a low scale $\left(\mathrm{J}_{0}\right)$, and multiple series of wavelet coefficients at scales higher than $\mathrm{J}_{0}$. The approximation coefficients reflect the low-frequency components with broad supports of the signal whereas the wavelet coefficients reflect the high-frequency components with compact supports of the signal. The approximation coefficient can be described as an inner product between the signal and a discrete, scaled, and shifted scaling function, and the wavelet 
coefficient can be described as an inner product between the signal and a discrete, scaled, and shifted wavelet function, as follows (Ge et al., 2007).

$$
\begin{aligned}
& A(j, k)=\left\langle f(n), \phi_{j k}(n)\right\rangle=\sum_{n=0}^{N-1} f(n) \times \phi_{j k}^{*}(n) \\
& D(j, k)=\left\langle f(n), \varphi_{j, k}(n)\right\rangle=\sum_{n=0}^{N-1} f(n) \times \varphi_{j, k}^{*}(n)
\end{aligned}
$$

where, $A(j, k)$ is an approximation coefficient, $D(j, k)$ is a wavelet coefficient, $<>$ denotes the inner product operation, * denotes a complex conjugate, and $f(n)$ is a signal with finite length of $n$.

Equations (3) and (4) represent the scaled and shifted versions of a discrete scaling function $\phi(n)$ and a wavelet function $\varphi(n)$, respectively. $S_{0}$ equals number two which represents dyadic discrete wavelet transform and $j$ and $k$ are the discrete versions of the scaling and shifting parameters, respectively.

$$
\begin{aligned}
& \phi_{j, k}(n)=s_{0}^{j / 2} \phi\left(s_{0}^{j} \cdot n-k\right) \\
& \varphi_{j, k}(n)=s_{0}^{j / 2} \varphi\left(s_{0}^{j} \cdot n-k\right)
\end{aligned}
$$

Practically, the DWT is implemented in a computationally efficient manner via the dyadic filter bank algorithm. This successive decomposition procedure is graphically illustrated in Figure 1, where $G$ and $H$ represent high-pass and low-pass digital filters, respectively. $A$ and $D$ represent the approximation coefficient and wavelet coefficient, respectively. The process starts from the original signal itself, and the signal is decomposed into a series of approximation coefficients and wavelet coefficients at a scale one level below. The resultant approximation series can be further decomposed into the approximation and wavelet series. This process can be repeated until it reaches at a specific scale, which is usually determined by the characteristics of the signal and the nature of the problem (Burrus et al., 1998).

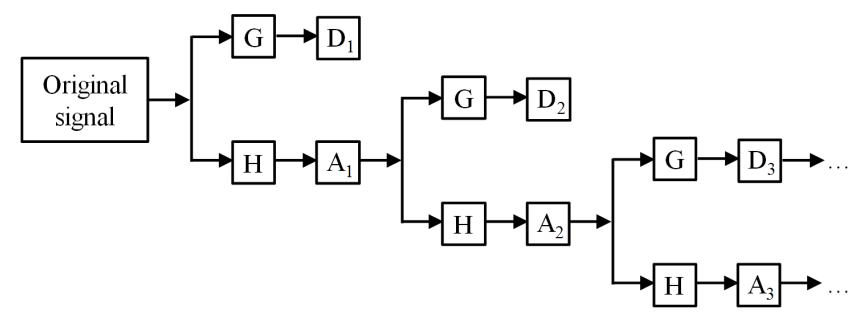

Figure 1. Successive dyadic filter bank algorithm used in DWT.
From equations (1) and (2), the inner product of the spectroscopic signal $f(n)$ and wavelet bases $\phi_{j, k}(n)$ or $\varphi$ $j, k(n)$ can be understood as a procedure for projecting the signal onto a set of orthogonal, complete, and compactly supported bases. Therefore, the resulting wavelet coefficients lose no information and are orthogonal to each other. In addition, this process enables the original signal which highly correlated spectroscopic wavebands can be decorrelated, and can also successfully solve the multicollinearity issue in conventional regression analysis. The multi-resolution capability of the DWT can be achieved by using the set of scaled and shifted versions of a wavelet function, as shown in equations (3) and (4), respectively, when spectroscopic data contain the overlapped spectra of different bandwidths centered at different wavelengths. In general, a good DWT wavelet coefficient can be obtained from a good match between a spectra and a wavelet base function. A good match implies the coincidence of central wavelengths and similarity between their spectral bandwidths, as well as the resemblance between the curves of a spectral signal and that of a wavelet base function. Hence, the resultant wavelet coefficients may be conducive to the physical interpretation of regression models when potential spectra are separated and identified not only by their central wavelengths but also by their spectral bandwidths. Thus, similarity in terms of shape such as depth, width, or asymmetry between a spectral signal and a wavelet base function should be carefully considered when a DWT is used in preprocessing.

We applied the DWT to measured transmittance spectra to generate new variables in the wavelet domain, which replaced the original spectral signal for subsequent PLS analysis and prediction modeling. The approximation coefficient series $A$ was used instead of the wavelet coefficient series $D$. Each cherry tomato transmittance spectrum was subject to six levels of dyadic filter bank decomposition, as shown in Figure 1, and approximation coefficient series from scales 2 to 4 were applied for subsequent PLS analysis and prediction modeling. This selection of scales was based on the fact that the bandwidths of significant transmittance spectra between 580 and $680 \mathrm{~nm}$ were observed to be less than $100 \mathrm{~nm}$, as shown in Figure 2. A spectral signal can be approximated with the Gaussian distribution, and the relationship between the bandwidth and full width at half maximum (FWHM) of a spectral signal can be expressed as equation (5) (Singer, 1981; Clark, 1981). The bandwidths of three 
applied scales (A2, A3, A4) correspond to 170,85 , and 42 $\mathrm{nm}$, respectively, which are equivalent to FWHMs of 72.2, 36.1 , and $17.8 \mathrm{~nm}$, respectively, as given by equation (5). Therefore, the selected approximation coefficients (A2, A3, A4) were expected to include most of the meaningful transmittances spectral features of cherry tomatoes. It is also known that noise can be dominant at a higher scale than A6 and that several spectral signals would overlap at a lower scale than A2. Therefore, the selection of scales from 2 to 4 was deemed to be appropriate in our study. The DWT was applied using the Wavelet Toolbox in commercial software MATLAB (ver. 8.0, MathWorks, USA), and the $\mathrm{db} 5$ wavelet base was used as the mother wavelet function.

$$
W=2 \times(2 \times \ln 2)^{1 / 2} \times \sigma
$$

where, $W$ is the bandwidth and $\sigma$ is the FWHM.

\section{The samples and measurements}

We collected one hundred-eighty cherry tomatoes from an orchard in May 2010. For each experiment, thirty cherry tomatoes were tested using VIS/NIRS (USB4000, Ocean Optics Inc., USA), the schematic of which is shown in Figure 3. Each sample was scanned eight times, and spectroscopic transmittance measurements were obtained by averaging the eight raw scans. A $50 \mathrm{~W}$ halogen lamp was used for the light source. This spectroscope operates at a high sensitivity range of 471 to $1154 \mathrm{~nm}$, and the spectrum resolution is $0.2 \mathrm{~nm}$. After acquiring the transmittance spectrum of each specimen, firmness, sugar content, and titratable acidity were measured using a universal testing machine (SY-T-001, Sunyoung Systech Co., Korea), refractometer (PR-32 $\alpha$, ATAGO, Japan), and pH meter (3-star bench-top, Thermorion, USA), respectively. Maximum, minimum, average and standard deviation of measurements for firmness, sugar content, and titratable acidity are listed in Table 1 . The transmittance spectral curves of whole cherry tomatoes are shown in Figure 2. An increase in transmittance was observed from 580 to $680 \mathrm{~nm}$ (visible region), with a maximum at approximately $640 \mathrm{~nm}$, where $680 \mathrm{~nm}$ is the red end of the visible spectrum. High transmittance in this region can be the indicative of pigments such as lycopene and carotenes varies significantly with ripening and are mainly responsible for the red color of the fruit. More detailed information on firmness, sugar content, and
Table 1. Maximum, minimum, average and standard deviation of measurements for firmness, sugar content, and titratable acidity

\begin{tabular}{lccccc}
\multicolumn{1}{c}{ Measurements } & Maximum & Minimum & Average & $\begin{array}{c}\text { Standard } \\
\text { deviation }\end{array}$ \\
\hline Firmness $\left(\mathrm{kg}_{\mathrm{f}}\right)$ & 27.17 & 4.76 & 12.26 & 5.28 \\
\hline Sugar content $\left({ }^{\circ}\right.$ Brix) & 8.90 & 4.20 & 6.03 & 0.92 \\
\hline Titratble acidity $(\%)$ & 1.93 & 0.58 & 1.26 & 0.34 \\
\hline
\end{tabular}

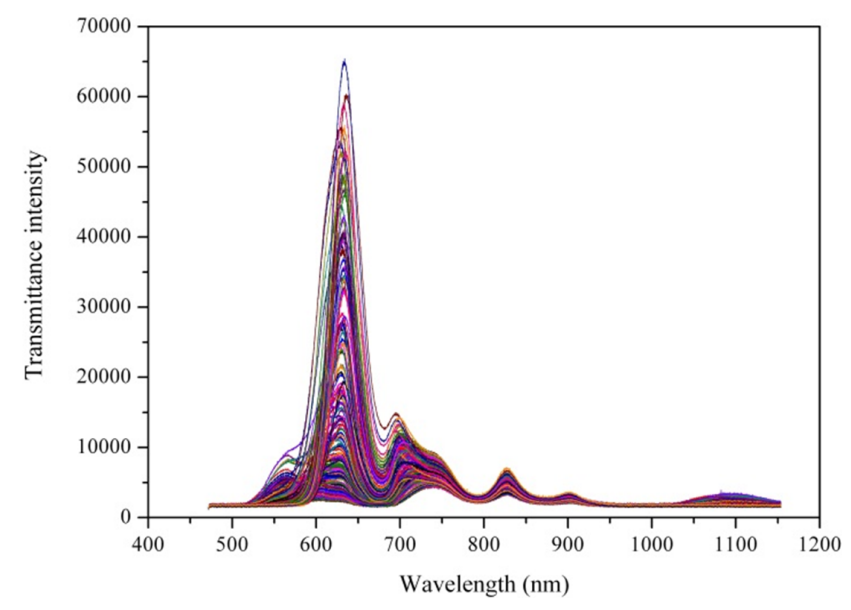

Figure 2. Transmittance spectral curves of cherry tomatoes obtained using VIS/NIRS.

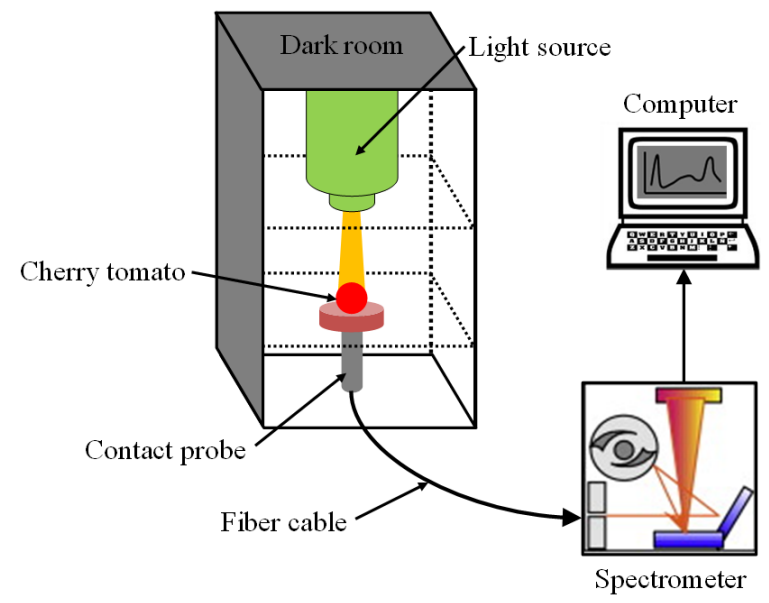

Figure 3. Schematics of VIS/NIRS instruments used in tests.

titratable acidity measurements of cherry tomato samples are reported by Kim (Kim et al., 2010).

PLS analysis was used to develop calibration and prediction models for the firmness, sugar content, and titratable acidity of cherry tomatoes. We used one hundred twenty randomly selected cherry tomato samples to construct calibration models, and the remaining 60 samples were used for evaluating the prediction performances. PLS analysis was carried out using the commercial soft- 
ware Unscrambler (ver. 9.1, CAMO, Norway), a statistical software package for multivariate calibration. A DWT preprocessing-incorporated PLS model, nonpreprocessing PLS model, and mean normalization-preprocessed PLS model were developed and compared with respect to their coefficients of determination $\left(\mathrm{R}^{2}\right)$ and root mean squared errors (RMSEs) (Shao et al., 2007).

\section{Results and Discussion}

The $\mathrm{R}^{2}$ values and RMSEs of the calibration models for firmness, sugar content, and titratable acidity are listed in Table 2. In the case of firmness, the $\mathrm{R}^{2}$ of the DWTincorporated PLS model was 0.853 , which is higher than that of the PLS model that used raw data (0.834) and slightly lower than that of the mean normalized PLS model (0.875). However, in the case of sugar content, the DWT-incorporated PLS model yielded a higher $\mathrm{R}^{2}$ value (0.865) than the mean normalized PLS model (0.833) and a lower RMSE $\left(0.3381^{\circ} \mathrm{Brix}\right)$ than the mean normalized PLS model ( $0.376^{\circ}$ Brix). In the case of titratable acidity, the DWT-incorporated PLS model yielded a higher $\mathrm{R}^{2}$ value $(0.700)$ than both PLS models that used raw data (0.627) and mean normalization preprocessing (0.640), and the DWT-incorporated PLS model yielded a lower RMSE $(0.1832 \%)$ than both PLS models that used raw data $(0.2056 \%)$ and mean normalization preprocessing (0.202\%).

Table 3 compares the $\mathrm{R}^{2}$ values and RMSEs of the DWT-

Table 2. Results of PLS calibration models and full cross-validation for firmness, sugar content, and titratable acidity, which used raw data, mean normalization preprocessing, and DWT preprocessing methods

\begin{tabular}{|c|c|c|c|c|c|c|c|c|c|c|c|c|}
\hline \multirow{3}{*}{ Preprocessing } & \multicolumn{4}{|c|}{ Firmness (kgf) } & \multicolumn{4}{|c|}{ Sugar content ( ${ }^{\circ}$ Brix) } & \multicolumn{4}{|c|}{ Titratable acidity (\%) } \\
\hline & \multicolumn{2}{|c|}{ Cal } & \multicolumn{2}{|c|}{ Val } & \multicolumn{2}{|c|}{ Cal } & \multicolumn{2}{|c|}{ Val } & \multicolumn{2}{|c|}{ Cal } & \multicolumn{2}{|c|}{ Val } \\
\hline & $\mathrm{R}_{\mathrm{c}}{ }^{2}$ & RMSE & $\mathrm{R}_{\mathrm{v}}{ }^{2}$ & RMSE & $\mathrm{R}_{\mathrm{c}}{ }^{2}$ & RMSE & $\mathrm{R}_{\mathrm{v}}{ }^{2}$ & RMSE & $\mathrm{R}_{\mathrm{c}}{ }^{2}$ & RMSE & $\mathrm{R}_{\mathrm{v}}{ }^{2}$ & RMSE \\
\hline Raw data & 0.834 & 2.1532 & 0.808 & 2.3355 & 0.867 & 0.3357 & 0.753 & 0.4603 & 0.627 & 0.2056 & 0.620 & 0.2091 \\
\hline Normalization (mean) & 0.875 & 1.8669 & 0.849 & 2.0736 & 0.833 & 0.3760 & 0.708 & 0.5008 & 0.640 & 0.2020 & 0.634 & 0.2052 \\
\hline DWT & 0.853 & 2.0169 & 0.824 & 2.2152 & 0.865 & 0.3381 & 0.762 & 0.4554 & 0.700 & 0.1832 & 0.661 & 0.1949 \\
\hline
\end{tabular}

Table 3. Results of PLS prediction models for firmness, sugar content, and titratable acidity, which used raw data, mean normalization preprocessing, and DWT preprocessing methods

\begin{tabular}{|c|c|c|c|c|c|c|c|c|c|}
\hline \multirow{2}{*}{ Preprocessing } & \multirow{2}{*}{ factor } & \multicolumn{2}{|c|}{ Firmness (kgf) } & \multirow{2}{*}{ factor } & \multicolumn{2}{|c|}{ Sugar content ( ${ }^{\circ}$ Brix) } & \multirow{2}{*}{ factor } & \multicolumn{2}{|c|}{ Titratable acidity (\%) } \\
\hline & & $\mathrm{R}_{\mathrm{p}}^{2}$ & RMSE & & $R_{p}^{2}$ & RMSE & & $R_{p}^{2}$ & RMSE \\
\hline Raw data & 7 & 0.860 & 1.8828 & 11 & 0.820 & 0.4001 & 1 & 0.442 & 0.2465 \\
\hline Normalization (mean) & 7 & 0.874 & 1.7945 & 9 & 0.869 & 0.3423 & 1 & 0.486 & 0.2365 \\
\hline DWT & 9 & 0.884 & 1.7096 & 18 & 0.818 & 0.3950 & 7 & 0.545 & 0.2208 \\
\hline
\end{tabular}

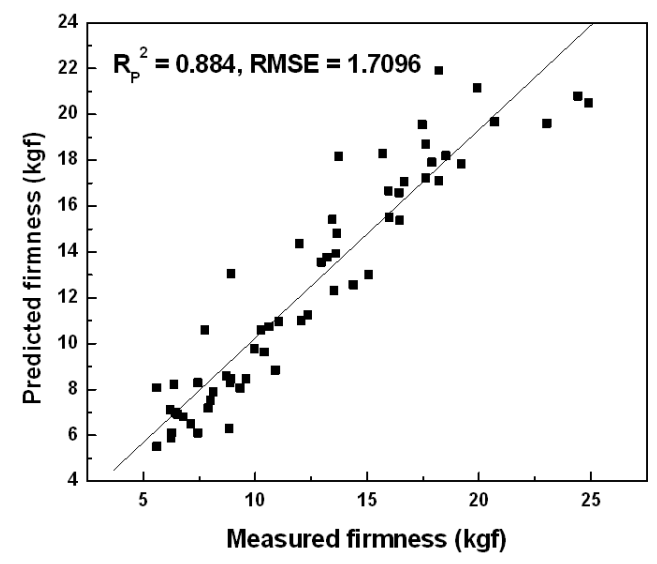

(a)

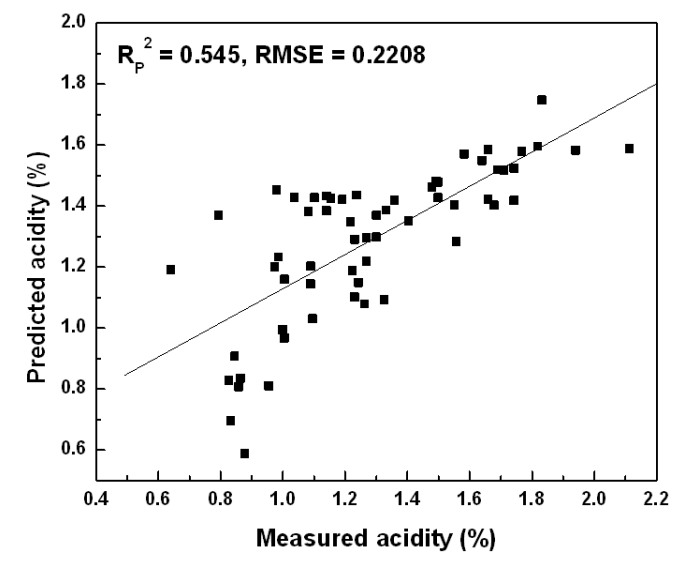

(b)

Figure 4. Comparison of DWT-incorporated PLS model versus measured firmness (a) and titratable acidity $(b)$ in prediction $(n=60)$. 
incorporated PLS prediction models with those of the PLS models that used raw data and mean normalization preprocessed datasets for firmness, sugar content, and titratable acidity. In terms of prediction capability, the DWT-incorporated PLS model yielded the highest $\mathrm{R}^{2}$ values of 0.884 and 0.545 and the lowest RMSEs of 1.7096 and 0.2208 for firmness and titratable acidity, respectively. However, the $\mathrm{R}^{2}$ value (0.818) and RMSE (0.3950) of the DWT-incorporated PLS model for sugar content was similar to the $\mathrm{R}^{2}$ value $(0.820)$ and RMSE $(0.4001)$ of the PLS model that used raw data. Figure 4 shows scatter plots comparing the measured firmness and titratable acidity with the values predicted by the DWT-incorporated PLS model.

\section{Conclusions}

We incorporated a DWT as a preprocessing tool to spectral data obtained from VIS/NIRS. The developed DWT-incorporated PLS models using the db5 wavelet base and selected approximation coefficients showed improved prediction results for the firmness and titratable acidity of cherry tomatoes with respect to $\mathrm{R}^{2}$ values and RMSEs. The reasons why the DWT-incorporated PLS method performs better than the PLS method that uses raw data and better than conventional preprocess methods are given in other studies. Most of these emphasize the fine- and coarse-scale separation of the spectral features and the reduction of the dimensionality of spectroscopic data. Thus, new variables may be decorrelated with original spectral information without the loss of spectral information.

In our research, our DWT-incorporated PLS model showed improvements in predicting the internal quality of cherry tomatoes by accurately selecting the wavelet base and transform scales. However, it is important to note that the prediction performance of PLS analysis for VIS/NIR spectra can vary according to the applied transform scales and wavelet base. The reasons for these variations should be considered, and additional research is therefore necessary to find optimal methods of selecting appropriate wavelet base and transform scales when using a DWT as a preprocessing tool for VIS/NIR spectra.

\section{Conflict of Interest}

No potential conflict of interest relevant to this article was reported.

\section{Acknowledgement}

This study was partially supported by the Korea Basic Science Institute Grant (D32200) and Technology Development Program for Agriculture and Forestry, Ministry for Food, Agriculture, Forestry and Fisheries, Republic of Korea.

\section{References}

Arnold, M. A. and G. W. Small. 1990. Determination of physiological levels of glucose in an aqueuos matrix with digitally filtered fourier transform near-infrared spectra. Analytical Chemistry 62(14):1457-1464.

Brereton, R. G. 2000. Introduction to multivariate calibration in analytical chemistry. Analyst 125:2125-2154.

Bruce, L. M., C. H. Koger and J. Li. 2002. Dimensionality reduction of hyperspectral data using discrete wavelet transform feature extraction. IEEE Transactions on Geoscience and Remote Sensing 40(10):2331-2338.

Burrus, C. S, R. A. Gopinath and H. Guo. 1998. Introduction to wavelets and wavelet transforms: A Primer. Upper Saddle River, N.J.: Prentice-Hall.

Clark, R. N. 1981. Water frost and ice: The near-infrared spectral reflectance 0.65-2.5 $\mu \mathrm{m}$. Journal of Geophysical Research:Solid Earch 86(V4):3087-3096.

Ge, Y., C. L. S. Morgan, J. A. Thomasson and T. Waiser. 2007. A new perspective to near-infrared reflectance spectroscopy: A wavelet approach. Transactions of the ASABE 50(1):303-311.

Jetter, K., U. Depczynski, K. Molt and A. Niemöller. 2000. Principles and applications of wavelet transformation to chemometrics. Analytica Chimica Acta 420(12): 169-180.

Karstang, T. V. and O. M. Kvalheim. 1991. Multivariate prediction and background correction using local modeling and derivative spectroscopy. Analytical Chemistry 63(8):767-772.

Kim, D. Y., B. Cho, C. Mo and Y. S. Kim. 2010 Study on prediction of internal quality of cherry tomato using 
Vis/NIR spectroscopy. Journal of Biosystems Engineering 35(6): 450-457 (In Korean, with English abstract).

McNulty, C. S. and G. R. Mauze. 1998. Application of wavelet analysis for determining glucose concentrations of aqueous solutions using NIR spectroscopy. In: Proceedings of SPIE, Infrared Spectroscopy: New Tool in Medicine, pp. 167-176, San Jose, CA.

Qi, X. M., L. D. Zhang and L. N. Chai. 1999. Study of nearinfrared spectra for quantitative analysis using principal component-stepwise regression analysis. Journal of Beijing Agricultural College 14:45-49.

Singer, R. B. 1981. Near-infrared spectral reflectance of mineral mixtures: Systematic combinations of pyroxenes, olivine, and iron oxides. Journal of Geophysical Research 86(B9): 7967-7982.
Shao, Y., Y. He, A. H. Gomez, A. G. Pereir, Z. Qiu and Y. Zhang. 2007. Visible/near infrared spectrometric technique for nondestructive assessment of tomato 'Heatwave' (lycopersicum esculentum) quality characteristics. Journal of Food Engineering 81(4): 672-678.

Svensson, O., T. Kourti and J. F. MacGregor. 2002. An investigation of orthogonal signal correction algorithms and their characteristics. Journal of Chemometrics 16(4):176-188.

Walczak, B., D. and L. Massart. 1997. Wavelets - something for analytical chemistry? TrAC Trends in Analytical Chemistry 16(8):451-463.

Zhang, X., N. H. Younan and C. G. O'Hara. 2005. Wavelet domain statistical hyperspectral soil texture classification. IEEE Trans. Geoscience and Remote Sensing 43(3): 615-618. 